\title{
Transient slider-disk contacts in the presence of spherical contamination particles
}

\author{
A. Ovcharenko $\cdot$ M. Yang • \\ K. Chun $\cdot$ F. E. Talke
}

Received: 20 July 2010/ Accepted: 29 December 2010/Published online: 15 January 2011

(C) The Author(s) 2011. This article is published with open access at Springerlink.com

\begin{abstract}
Transient thermal-mechanical contacts between a slider and a rotating disk in the presence of contamination particles are investigated using finite element analysis. The maximum temperature rise due to frictional heating, the maximum plastically deformed area, and the maximum residual penetration into the disk surface are determined as a function of particle properties for aluminum and glass disk substrate. It is found that contact with soft and deformable particles can cause a large temperature rise, sufficient for thermal erasure, even though physical damage of the media is not observed. Contact with hard and rigid particles can lead to severe plastic deformation as well as high temperature rise of the media, representing the worst case for tribological integrity of the slider-disk interface.
\end{abstract}

\section{Introduction}

The presence of contamination particles in a hard disk drive can lead to particle induced failure modes causing physical damage or thermal erasure of the recording media (Brand et al. 2007). Contamination particles inside an operating hard disk drive can result from assembly of the disk drive, from sliding of the suspension lift tab on the load-unload ramp, from fretting wear of the dimple/

\footnotetext{
A. Ovcharenko $(\square) \cdot$ M. Yang $\cdot$ K. Chun

Western Digital Corporation, San Jose, CA 95138, USA

e-mail: andreyo78@gmail.com
}

A. Ovcharenko - F. E. Talke

Center for Magnetic Recording Research,

University of California San Diego, La Jolla,

CA 92093, USA gimbal interface, or from slider disk contacts, to name a few. A longer list of potential sources of contamination particles in a hard disk drive is discussed by Zhang et al. (1999).

A number of investigations have been published on erasure of data and tribological failure of magnetic recording disk drives due to particles being trapped at the slider-disk interface. Zhang et al. (1999) studied experimentally the damaging effect of various contamination particles on the slider-disk interface. They found that particles can be embedded, deformed, or sheared during slider disk interactions, resulting in deformation and scratching of the disk surface. Xu et al. (2000) experimentally studied the presence of soft particles and proposed improved slider designs preventing the intrusion of soft particles in the slider-disk interface. A quasistatic model predicting scratch probability resulting from a hard particle trapped in the slider-disk interface was developed by Zeng et al. (2005). Roy and Brand (2007) experimentally investigated magnetic erasure of recording media due to soft particles. They reported that glass substrate media is more susceptible to thermal erasure than aluminum substrate media. No physical damage to the disk surface was observed. Shen and Bogy (2008) modeled motion of "large" elastic particles and showed that they tend to slide between slider and disk.

No studies have been published so far on the modeling of transient slider-disk contacts in the presence of contamination particles. The goal of the present work is to investigate this latter situation and study the tribological failure of the slider-disk interface by evaluating the temperature rise and the damage of the recording layer due to slider/disk contacts in the presence of spherical contamination particles for aluminum and glass substrate media. 


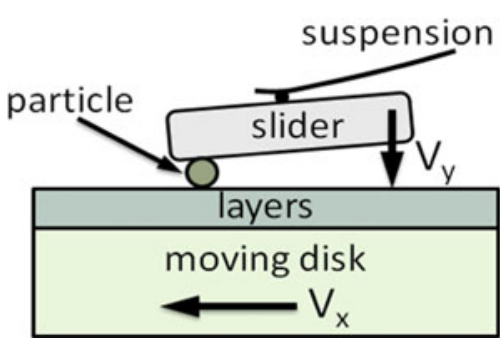

Fig. 1 Schematic model of a particle trapped in the slider-disk interface

\section{Modeling}

Figure 1 shows the schematic of the slider-disk interface during a transient contact in the presence of a single contamination particle. The impact velocity of the slider is denoted by $V_{\mathrm{y}}$ while the disk circumferential velocity is denoted by $V_{\mathrm{x}}$. The particle is assumed to be spherical in shape with radius $R$, adhering solidly to the air bearing surface of the slider during a transient contact. We also assume that the slider has a mass of $0.5 \mathrm{mg}$. Experimental studies conducted by Chung et al. (2004) and Kim et al. (2003) showed that contamination particles can adhere solidly to the slider air bearing surface and rub against the moving disk surface. This situation is typical for the loading process when the air bearing surface of a slider hits a contamination particle present on the disk surface. This situation is considered in the present study, unlike the case when a contamination particle enters the head/disk interface, e.g., Xu et al. (2000).

Figure 2 shows the finite element model of a particle/ disk contact for a disk with aluminum substrate. This model considers a multilayered disk structure for both aluminum and glass substrates as described by Ovcharenko et al. (2010). The spherical particle is assumed to have a constant initial temperature identical to that of the slider to which it adheres. Three materials were used to represent typical contamination particles, namely, stainless steel (SS), polyoxymethylene (POM), and alumina $\left(\mathrm{Al}_{2} \mathrm{O}_{3}\right)$. The mechanical and thermal material properties of these particles are summarized in Table 1. These contamination particles represent three groups of materials, namely, soft $(\mathrm{POM})$, semi-hard (SS), and hard $\left(\mathrm{Al}_{2} \mathrm{O}_{3}\right)$. The material properties and the schematic of the layered disk structure with aluminum and glass substrates are given by Ovcharenko et al. (2010).

\section{Results and discussion}

Figures 3, 4, and 5 show the maximum temperature rise, the plastically deformed area, and the maximum residual penetration on the disk surface, respectively, for stainless steel, polyoxymethylene and alumina contamination particles as a function of the particle radius for both glass and aluminum substrate disks. The numerical results were obtained for typical impact conditions of a slider on a disk, i.e., a slider vertical initial velocity of $V_{\mathrm{y}}=0.2 \mathrm{~m} / \mathrm{s}$, a disk circumferential velocity of $V_{\mathrm{x}}=10 \mathrm{~m} / \mathrm{s}$ and a coefficient of friction of $\mu=0.2$. These assumed values of the vertical initial velocity and the coefficient of friction correspond to typical cases observed experimentally by, e.g., Fu and Bogy (2000); Liu and Ma (2003); Kato et al. (2004).

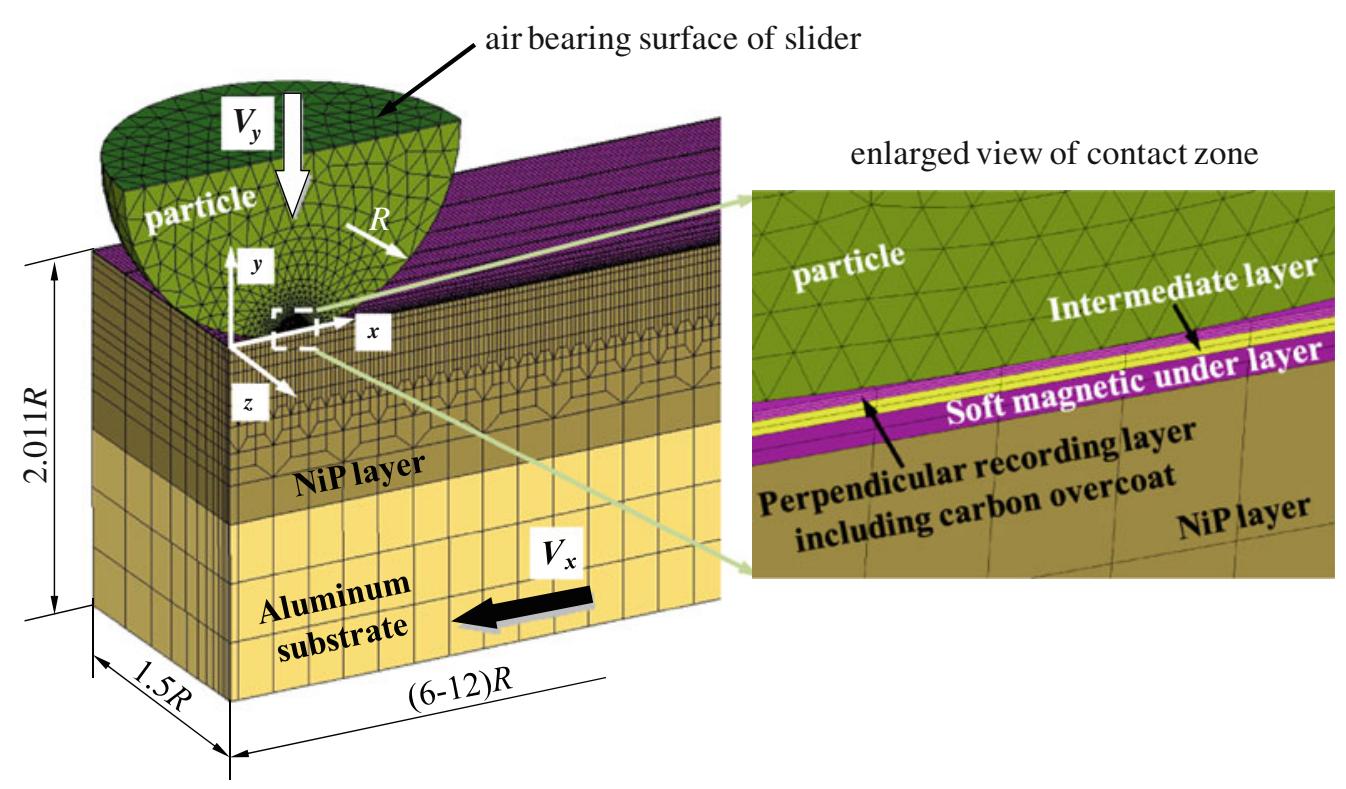

Fig. 2 Finite element model of contact between particle and layered disk with aluminum substrate 
Table 1 Material properties of contamination particles: stainless steel (SS), polyoxymethylene (POM), alumina $\left(\mathrm{Al}_{2} \mathrm{O}_{3}\right)$

\begin{tabular}{lllll}
\hline Property & Symbol (units) & POM & $\mathrm{SS}$ & $\mathrm{Al}_{2} \mathrm{O}_{3}$ \\
\hline Young's modulus & $E(\mathrm{GPa})$ & 3 & 195 & 400.0 \\
Yield strength & $Y(\mathrm{GPa})$ & 0.03 & 0.48 & 6.40 \\
Poisson's ratio & $v(-)$ & 0.40 & 0.276 & 0.30 \\
Density & $\rho\left(\mathrm{kg} \mathrm{m}^{-3}\right)$ & 1,450 & 7,840 & 4,300 \\
Specific heat & $c\left[\mathrm{~J}\left(\mathrm{~kg} \mathrm{~K}^{-1}\right]\right.$ & 1,470 & 498 & 860 \\
Thermal conductivity & $k\left[\mathrm{~W}\left(\mathrm{~m} \mathrm{~K}^{-1}\right]\right.$ & 0.21 & 15.20 & 24.00 \\
Thermal expansion & $\alpha\left(10^{-6} \mathrm{~K}^{-1}\right)$ & 60.0 & 17.6 & 7.5 \\
\hline
\end{tabular}

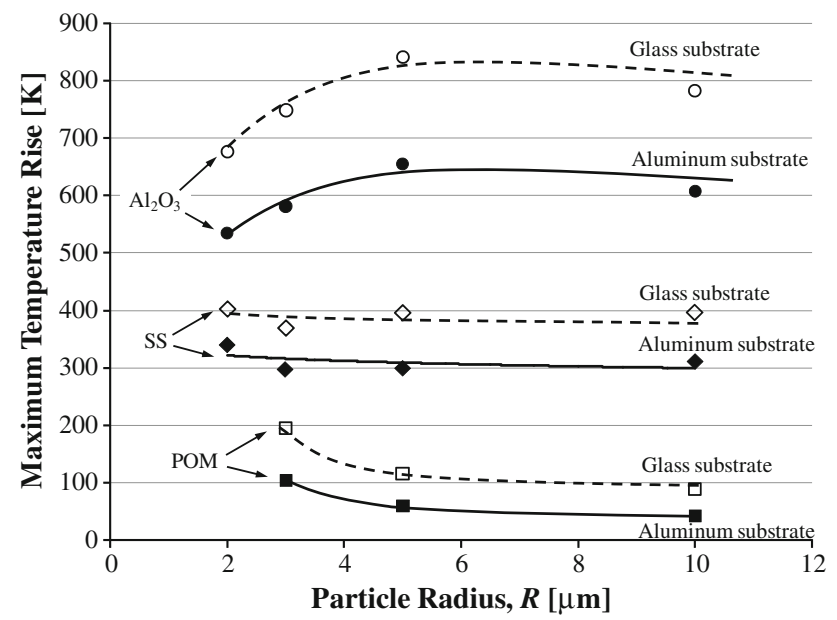

Fig. 3 Maximum temperature rise on the surface of the recording layer caused by contamination particles for glass and aluminum substrate $\left(V_{\mathrm{y}}=0.2 \mathrm{~m} / \mathrm{s}, V_{\mathrm{x}}=10 \mathrm{~m} / \mathrm{s}, \mu=0.2\right)$

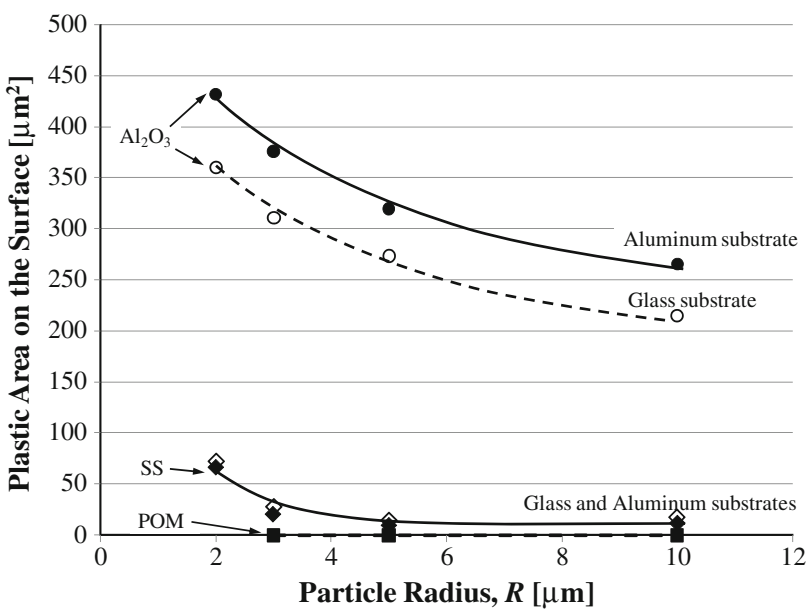

Fig. 4 Plastic area on the surface of the recording layer caused by contamination particles for glass and aluminum substrate $\left(V_{\mathrm{y}}=\right.$ $0.2 \mathrm{~m} / \mathrm{s}, V_{\mathrm{x}}=10 \mathrm{~m} / \mathrm{s}, \mu=0.2$ )

Figure 3 shows the maximum temperature rise as a function of particle radius. We observe from Fig. 3 that glass substrate disks result in a higher maximum

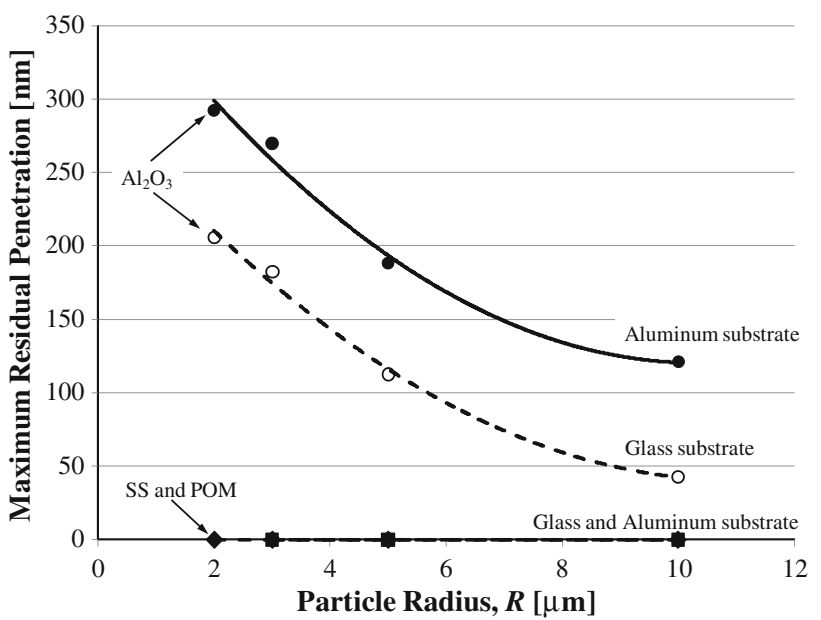

Fig. 5 Maximum residual penetration on disk surface caused by contamination particles for glass and aluminum substrate $\left(V_{\mathrm{y}}=\right.$ $0.2 \mathrm{~m} / \mathrm{s}, V_{\mathrm{x}}=10 \mathrm{~m} / \mathrm{s}, \mu=0.2$ )

temperature rise in comparison with $\mathrm{Ni}-\mathrm{P} /$ aluminum substrate, which is consistent with findings reported by Ovcharenko et al. (2010). Roy and Brand (2007) reported that media with glass substrate is more susceptible to thermal erasure induced by a soft particle than media with aluminum substrate. This finding is in line with the numerical results in Fig. 3 for POM particles showing a higher temperature rise on the surface of the recording layer for the glass substrate in comparison with the aluminum substrate due to its lower thermal conductivity. We observe that alumina particles lead to a higher maximum temperature rise than semi-hard stainless steel or soft polyoxymethylene particles due to the larger contact pressure resulting in higher frictional heating. We also observe that the maximum temperature rise is a function of the particle radius for small radii and becomes independent of particle size for particle radii larger than $5 \mu \mathrm{m}$.

Figure 4 shows that for hard alumina particles, glass substrate results in a smaller plastically deformed area in comparison with aluminum substrate due to the higher yield strength of glass. However, for the softer stainless steel contamination particles we observe nearly the same values of the plastically deformed area for both substrates. We also note that soft polyoxymethylene particles do not cause any plastic deformation (physical damage) on the surface of the recording layer. The plastic area on the surface of the recording layer tends to decrease with larger particle radius since contact pressure decreases.

Figure 5 shows the maximum residual penetration as a function of particle radius. We observe that the maximum residual penetration is larger for aluminum substrate than for glass substrate. The latter is in agreement with the numerical results shown in Fig. 4. For the numerical conditions assumed, semi-hard stainless steel and soft 
Fig. 6 Temperature distribution and deformation of the contact region for a alumina, b stainless steel,

c polyoxymethylene particles at the moment of maximum contact force (aluminum substrate disk, $V_{\mathrm{y}}=0.2 \mathrm{~m} / \mathrm{s}$, $V_{\mathrm{x}}=10 \mathrm{~m} / \mathrm{s}, \mu=0.2$, and $R=3 \mu \mathrm{m})$ (a)

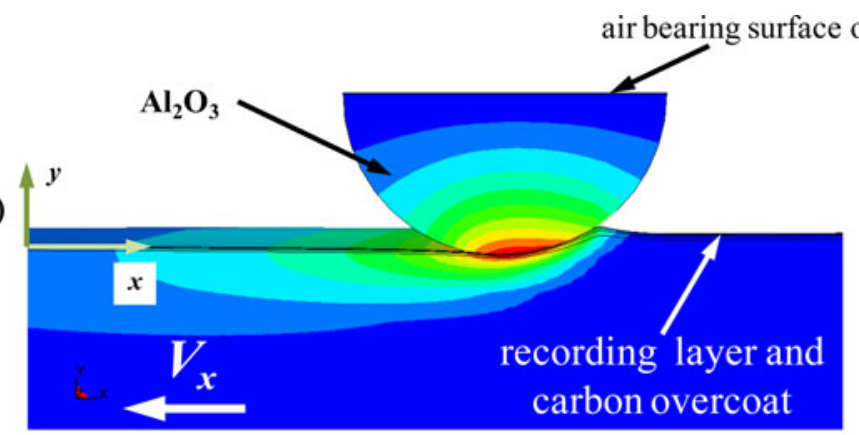

Temperature $[\mathrm{K}]$

(b)

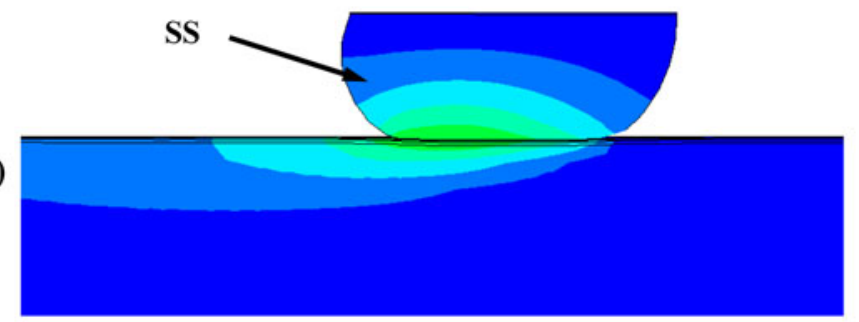

POM

(c)

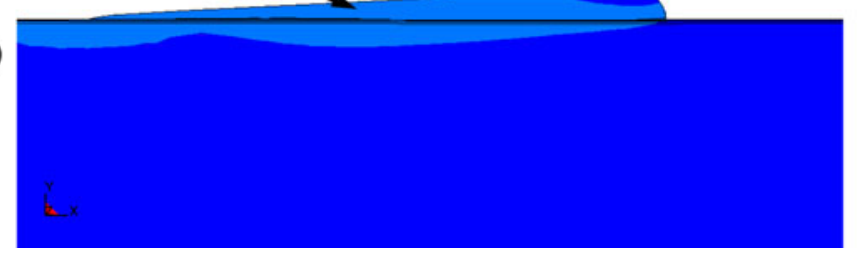

$8.700 \mathrm{e}+02$

$8.130 \mathrm{e}+02$

$7.560 \mathrm{e}+02$

$6.990 \mathrm{e}+02$

$6.420 \mathrm{e}+02$

$5.850 \mathrm{e}+02$

$5.280 \mathrm{e}+02$

$4.710 \mathrm{e}+02$

$4.140 \mathrm{e}+02$

$3.570 \mathrm{e}+02$

$3.000 \mathrm{e}+02$ polyoxymethylene particles do not result in any residual penetration on the disk surface for either substrate. The numerical results in Fig. 5 are consistent with experimental observations by Roy and Brand (2007) concerning the absence of physical damage of the media surface in the presence of soft particles and results by Zhang et al. (1999) concerning scratches (physical damage) in the case of hard particles. We also observe that larger hard particles lead to shallow residual deformations on the disk surface due to lower contact pressure leading to smaller penetration.

Figure 6 shows temperature distribution and deformation of the contact region for alumina, stainless steel and polyoxymethylene particles at the moment of maximum contact force. The results are shown for an aluminum substrate disk with $V_{\mathrm{y}}=0.2 \mathrm{~m} / \mathrm{s}, V_{\mathrm{x}}=10 \mathrm{~m} / \mathrm{s}, \mu=0.2$, and $R=3 \mu \mathrm{m}$. We observe from Fig. $6 \mathrm{a}$ that hard and rigid alumina particles do not undergo deformation and penetrate into the media causing severe deformation and a high temperature rise. Figure $6 \mathrm{~b}$ shows that semi-hard stainless steel particles cause negligible residual deformation on the media surface, but result still in a high temperature rise. Figure $6 \mathrm{c}$ shows the results for a soft polyoxymethylene particle. This case causes only a small temperature rise with no deformation of the media. From Fig. 6 we observe that hard particles such as alumina represent the most severe situation leading to physical damage of the media and a very high temperature rise. This can cause demagnetization in a way similar to slider-disk contacts as studied by Ovcharenko et al. (2010).

Numerical simulations of particles with radii $<2 \mu \mathrm{m}$ were also performed. Depending on the material properties of the contamination particles, we observed that small particles are either pushed deep into the substrate (alumina) or are flattened out on the surface of the disk (stainless steel and polyoxymethylene).

\section{Conclusions}

The slider-disk interface in the presence of contamination particles with different material properties and radii was studied for media with glass and aluminum substrates. From the numerical analysis and the results obtained we can conclude

1. Soft particles such as polyoxymethylene particles do not show residual plastic deformation of the magnetic recording layer during transient slider disk contacts. Temperature increases on the order of $100^{\circ}$ of Centigrade are predicted, indicating that thermal erasure may be a possible tribological failure mechanism.

2. Semi-hard particles such as stainless steel particles seem to cause little physical damage (residual plastic 
deformation) of the recording layer, but result in a temperature rise of several hundred degrees of Centigrade, which could lead to thermal erasure.

3. Hard particles such as alumina particles are predicted to result in significant plastic deformation combined with high temperature rise which can lead to loss of data in a similar way as "conventional" slider-disk contacts. It is apparent that hard contamination particles represent the most severe problem for the tribological integrity of magnetic recording disk drives.

Open Access This article is distributed under the terms of the Creative Commons Attribution Noncommercial License which permits any noncommercial use, distribution, and reproduction in any medium, provided the original author(s) and source are credited.

\section{References}

Brand JL, Roy M, Frenz AD (2007) Designing, modeling, and testing particle robust air bearings for perpendicular recording media. IEEE Trans Magn 43(9):3791-3795

Chung K-H, Oh J-K, Moon J-T, Kim D-E (2004) Particle monitoring method using acoustic emission signal for analysis of slider/disk/ particle interaction. Tribol Int 37:849-857
Fu T-C, Bogy DB (2000) Analysis of stresses induced by dynamic load head-disk contacts. ASME J Tribol 122(1):233-237

Kato T, Kawaguchi M, Sajjad MM, Choi J (2004) Friction and durability characteristics of ultrathin perfluoropolyether lubricant film composed of bonded and mobile molecular layers on diamond-like carbon surfaces. Wear 257(9-10):909-915

Kim D-E, Chung K-H, Cha K-H (2003) Tribological design methods for minimum surface damage of HDD slider. Tribol Int $36: 467-473$

Liu B, Ma YS (2003) Visualization and characterization of slider-disk interactions in dynamic load/unload process. IEEE Trans Magn 39(2):743-748

Ovcharenko A, Yang M, Chun K, Talke FE (2010) Simulation of magnetic erasure due to transient slider/disk contacts. IEEE Trans Magn 46(3):770-777

Roy M, Brand JL (2007) Soft particle-induce magnetic erasure without physical damage to the media. ASME J Tribol 129:729734

Shen X, Bogy DB (2008) Contact force and frictional heating due to "large" particles in the head disk interface. ASME J Tribol 130:1-7

Xu J, Tokisue H, Kawakubo Y (2000) Study on soft-particle intrusion in a head/disk interface of load/unload drives. IEEE Trans Magn 36(5):2745-2747

Zeng Q, Pit R, Payne R, Baumgart P, Huang F-Y (2005) Modeling and simulation of hard-particle interaction in head/disk interfaces. IEEE Trans Magn 41(2):604-609

Zhang L, Koka R, Yuen Y, Lam E (1999) Particle induced damage on heads and discs due to fine particles of different materials. IEEE Trans Magn 35(2):927-932 